\title{
Von Rahmenlehrplänen zu Curricula
}

Biografieperspektivische Beobachtungen zu aktuellen Entwicklungsprozessen an Pflegeschulen

\section{Claudia Winter}

Die neuen pflegefachlichen und pflegepädagogischen Standards der generalistischen Rahmenlehrpläne fordern Lehrende bei der curricularen Ausgestaltung heraus. An ausgewählten Narrativen werden dadurch entstehende curriculare Verhinderungsmuster skizziert. Gleichzeitig lässt sich aus diesen Mustern heraus eine erfahrungsbasierte Anregung zur gemeinsamen curricularen Haltungsarbeit von Lehrenden formulieren.

\section{Durch Narrative in Pflegeschulen blicken}

Nach der Veröffentlichung der Rahmenlehrpläne der Fachkommission am 1. August 2019 begannen Pflegeschulen zur Vorbereitung auf die generalistische Pflegeausbildung Schulentwicklungsprozesse, um vor allem handlungsfähig zu werden. Lehrende an den Schulen versuchen seither die vorgelegten Rahmenlehrpläne in schulinternen Curricula abzubilden, damit sie möglichst gut darauf vorbereitet sind, neue Auszubildende zu unterrichten. Die dabei von mir begleiteten Prozesse sind nach meiner Wahrnehmung anspruchsvoll, schwierig zu planen und mühsam umsetzbar. Ausgehend von der These, dass viele Lehrende aufgrund eigener Erfahrungen und Gewohnheiten handeln, auch wenn sie sich mit didaktischen Theorien auseinandergesetzt hatten (Oelke i.E.), lassen sich insbesondere berufssoziologische Beobachtungen, die relevant sind für curriculare und zugleich wirklich generalistische Entwicklungsbestrebungen, in Narrativen bestätigen. Denn Narrative explizieren aufgrund ihrer „inhärente[n] Erklärungskraft" (Nünning 2011/2012, S. 91) Begründungszusammenhänge, konkretisieren Beziehungen, lassen Geschehenes verständlich werden und sind insofern sinnund bedeutungsstiftend, als sie die Gestaltung von biografisch geprägten Lernprozessen unterstützen können.

Vor dem Hintergrund dieser hermeneutischen Heuristik wurden im Sommersemester 2020 Studierende des Studiengangs Gesundheits- und Pflegepädagogik an der Evangelischen Hochschule Nürnberg in einer Lehrveranstaltung zur Curriculumentwicklung darum gebeten, herausfordernde Situationen während der Arbeit an schulinternen Curricula zu dokumentieren, die sie im Wintersemester 2019/2020 an Pflegeschulen während der Ableistung ihres Praxissemesters erlebten. Der Arbeitsauftrag lautete:

1. Erinnern Sie sich an eine oder mehrere Situationen, in denen Sie während Ihres Praxissemesters Einblicke in die curriculare Arbeit an Pflegeschulen erhalten haben.

2. Erstellen Sie zu einer dieser Situationen ein Narrativ, indem Sie die Situation sowie Ihre Eindrücke und Empfindungen beim Erleben der Situation beschreiben. 
Hinweis: Diese Artikelfassung entspricht nicht vollständig dem in der Zeitschrift PADUA veröffentlichten Artikel unter https://doi.org/10.1024/1861-6186/a000579. Dies ist nicht die Originalversion des Artikels und kann daher nicht zur Zitierung herangezogen werden. Bitte verbreiten oder zitieren Sie diesen Artikel nicht ohne Zustimmung des Autors.

3. Reflektieren Sie auch darüber, was Sie nach dem Erleben dieser Situation bewegte und wie Sie die Situation bewertet hatten.

In der Lehrveranstaltung sollten sich Studierende mit eigenen Erlebnissen insofern reflexiv auseinandersetzen, als sie diese einerseits aus pflegewissenschaftlicher und pflegedidaktischer Perspektive prüfen und andererseits aus dem eigenen Selbstverständnis heraus deuten. Auffällig ist, dass in den vorliegenden 15 Narrativen ausschließlich Erlebnisse zu curricularen Prozessen ,erzählt' werden, die durch Überforderung, Spannungen, Widerstände und Konflikte gekennzeichnet sind. Aufgrund typischer Ursachen, die Studierende beschreiben, lassen sich drei curriculare Verhinderungsmuster in den Narrativen identifizieren. Durch deren Erschließung könnten Lehrende sich dazu anregen lassen, über eigene aktuelle curriculare Prozesse nachzudenken.

\section{Erstes Verhinderungsmuster: Eine große Aufgabe}

Anfangs werden viele Lehrende überfordert von der Fülle der Rahmenlehrpläne. Sie fragen sich, ob sie auf Altbewährtes aufbauen können oder Neues entwickeln müssen. Viele wünschen sich eher Anknüpfungspunkte an bislang gehaltene Unterrichte sowie konkrete Umsetzungshinweise und praktikable Instrumente zur curricularen Ausarbeitung, als über grundlegende Begriffe diskutieren zu wollen.

Vielen Lehrenden fällt es schwer, die hohe Komplexität der Rahmenlehrpläne zu erfassen, denn mit innen wird ein Standard gesetzt, der sich vor allem charakterisieren lässt durch

- seine anspruchsvolle pflegedidaktische und pflegewissenschaftliche Ausrichtung,

- eine gleichsam riesige Stofffülle von über 300 Seiten,

- einem neuen Kompetenzbegriff und

- die Abkehr vom Lernfeldkonzept der KMK.

Darüber hinaus sind die im Begründungsrahmen der Rahmenlehrpläne skizzierten „wesentliche[n] Überzeugungen und Prinzipien“ (Fachkommission 2020a, S. 8), die für die Fachkommission entwicklungsleitend waren, nach meiner Wahrnehmung an vielen Stellen sehr ambitioniert und deshalb für Lehrende unklar und irritierend. Sie verwirren wohl auch, weil sie erst im Nachgang mit Begleitmaterialien versehen wurden, in denen die "grundlegende Überzeugung“ (Fachkommission 2020a, S. 9) der Fachkommission in Bezug auf das lehrplangemäße Pflege- und Berufsverständnis sowie didaktisch-pädagogische Grundsätze niedergelegt sind.

Vor diesem Hintergrund dürfte das folgende Narrativ entstanden sein:

„Der stellvertretende Leiter des Schulzentrums schien etwas verärgert über die geringe Teilnehmerzahl trotz der Wichtigkeit dieses Treffens. Zunächst stellte er die 
Hinweis: Diese Artikelfassung entspricht nicht vollständig dem in der Zeitschrift PADUA veröffentlichten Artikel unter https://doi.org/10.1024/1861-6186/a000579. Dies ist nicht die Originalversion des Artikels und kann daher nicht zur Zitierung herangezogen werden. Bitte verbreiten oder zitieren Sie diesen Artikel nicht ohne Zustimmung des Autors.

einzelnen curricularen Einheiten vor und erläuterte den Aufbau des Lehrplans. Ich hatte den Eindruck, dass er sich sehr engagiert mit dem Lehrplan auseinandergesetzt hat, wohingegen die Kollegen sich bis dahin wenig bis gar nicht mit dem Lehrplan beschäftigt haben. Gedacht war, dass bei diesem Treffen schon mit der Ausarbeitung der einzelnen CE begonnen wird. Beim Durchblättern der über 300 Seiten kam es immer wieder zu Verwirrungen und Fragen, sodass die geplante Ausarbeitung nicht möglich war. Der stellvertretende Leiter appellierte an seine Kollegen, dass es für die Ausarbeitung unabdingbar sei, sich den Lehrplan vollständig durchzulesen, um einen Überblick zu erhalten und anschließend zielführend damit arbeiten zu können. Er schien etwas frustriert und äußerte, dass er nicht die alleinige Verantwortung sowie die zeitliche Kapazität habe, diese große Aufgabe alleine zu bewerkstelligen. Insgesamt schien das Treffen auf mich frustrierend, da die Mitarbeiter eher lustlos wirkten. " (N_7)

\section{Interpretation}

Erstmals sind Lehrende dazu aufgefordert, schulinterne Curricula zu entwickeln, die nicht dem bekannten KMK-Lernfeldansatz folgen. Sie sind nicht vorbereitet auf den dazu fehlenden Bezug, der andere Anforderungen als bislang an die Pflegeausbildung stellt. Beunruhigend ist insbesondere, dass in Zeiten des Wandels gewohnte Muster pädagogischer Arbeit innerhalb organisationaler Grenzen, Strukturen und Regeln nicht mehr wirken. Wie neue Curricula zu entwickeln sind, können scheinbar weder der stellvertretende Schulleiter noch Lehrende situativ klären. Folglich fehlt der bisher Sicherheit vermittelnde organisatorische Rahmen. Lehrende spüren wohl, dass sie aktuell vor der Aufgabe stehen, nicht nur die eigene Profession, sondern auch die Pflegeschule als Organisation entwickeln zu müssen (Göhlich 2008, S. 264), und werden durch diese Erkenntnis überfordert und gehemmt.

In Zeiten des Wandels benötigen sie aber Sicherheit und Entlastung. Denn in den aktuellen generalistischen Veränderungsprozessen handeln sie nicht rational ziel- bzw. zweckorientiert, sondern um Bedürfnisse zu befriedigen, die sie unbefriedigt wiederum mit der (äußeren) Haltung - hier: Lustlosigkeit - sichtbar werden lassen (Göhlich 2008, S. 269). Panke-Kochinke geht davon aus, dass Prozesse erfolgreicher Schulentwicklung an Pflegeschulen nur gelingen, falls „die Komponente[n] der Sicherheitsvermittlung und Entlastung“ (2016, S. 89) bei allen Entscheidungen von Beginn an mitgedacht werden. Will demnach der stellvertretende Schuleiter Lehrende motivieren, sollte er einerseits nach Ausgangs- und Anknüpfungspunkten suchen, die sich an deren bisherigen Einstellungen und Haltungen orientieren, und andererseits curriculare „Interventionen beschränken auf wenige und zudem nicht zusätzlich belastende Elemente" (Panke-Kochinke 2016, S. 97).

Konzentrieren sich curriculare Entwicklungen auf Lehrende (und nicht auf das System Schule), betreffen sie Handlungen von Lehrenden - und eben nicht die Schule. Durch die Thematisierung pädagogischer Handlungsprobleme sind „Widersprüche, Paradoxien oder Antinomien“ (Reh 2004, S. 360) als Kernprobleme beruflicher Praxis bearbeitbar. Pädagogische Professionalität zeigen Lehrende insbesondere durch 
Hinweis: Diese Artikelfassung entspricht nicht vollständig dem in der Zeitschrift PADUA veröffentlichten Artikel unter https://doi.org/10.1024/1861-6186/a000579. Dies ist nicht die Originalversion des Artikels und kann daher nicht zur Zitierung herangezogen werden. Bitte verbreiten oder zitieren Sie diesen Artikel nicht ohne Zustimmung des Autors.

Selbstreflexion (Oevermann 1996, in: Reh 2004, S. 364): Sie klären ihren ,biografischen Standort', indem sie die eigenen biografisch entstandenen ,Geschichten' versprachlichen, um (wieder) handlungs- und reaktionsfähig zu werden (Reh 2004, S. 364). Den dafür notwendigen Rahmen hätte der stellvertretende Schulleiter wohl erst schaffen müssen.

\section{Zweites Verhinderungsmuster: Die Sachen klären}

Lehrende greifen in vielen Fällen zurück auf Erfahrungswissen, um damit (schnell oder wieder) handlungsfähig zu werden und erklären dementsprechende Konzepte aufgrund von Zeitknappheit schnell zu scheinbar geeigneten Instrumenten auf dem Weg zur Lösung. Ob bzw. wie allerdings diese Instrumente zu den Rahmenlehrplänen passen, wird nicht geprüft, sodass oft kein wirklich generalistischer Lernprozess beginnt.

Nicht alle Lehrenden verfügen über eine differenzierte Vorstellung darüber, wie sie neue Curricula unter systematischem Rückgriff auf (pflege-)didaktische Theorien, Modelle und Konzepte sowie orientiert an den Bedürfnissen der Schule, aber auch der Praxiseinrichtungen entwickeln sollen. Vielmehr gehen sie aus von ihren „biographische[n] Basisdispositionen“ (Ostermann-Vogt 2011, S. 13), d.h. bereits gemachte „Erfahrungen [bilden] die Basis für die weiteren Erfahrungen im Verlaufe des Lebens“ (Ostermann-Vogt 2011, S. 14). Viele Lehrende stehen also vor einer Aufgabe, die sie zwar bestenfalls - theoretisch - während der hochschulischen Lehrerqualifizierung kennen gelernt haben, aber niemals in praxi bewältigten. Oder sie hatten bereits Curricula entwickelt, allerdings ohne dabei den oben aufgezeigten Standard zu verfolgen. Folglich geraten sie in spannungsreiche und widersprüchliche Situationen, werden unsicher und ängstlich, sodass sie die Entwicklung von schulinternen Curricula entweder - wie im ersten Narrativ - gar nicht erst in Gang setzen oder aber misslingen lassen, weil sie ,alten Wein in neue Schläuche füllen'. Die Curriculumentwicklung wird zum ,Produkt' des lebensgeschichtlich gewordenen Professionsverständnisses Lehrender: Sie analysieren zwar die Rahmenlehrpläne aus ihren Erfahrungen heraus und aufgrund subjektiver Theorien und entwickeln schulinterne Curricula nach ihren bisherigen pflegeberuflichen und pädagogischen Werten, dürften sich aber dessen kaum bewusst sein (Ostermann-Vogt 2011, S. 250).

Entsprechende Theorien wirken wohl im folgenden Narrativ:

„Viele der Teilnehmer der Curriculum-AG sind schon viele Jahre an der Krankenpflegeschule und haben das vorherige Curriculum mitentwickelt. Es waren nun also viele Befürworter der Didaktik nach Karin Wittneben, da sie mit dieser schon seit den letzten 16 Jahren arbeiteten, sie kannten, verstanden und umzusetzen wussten. Jedoch gab es auch eine junge Lehrkraft, die erst seit einem Jahr im neuen Beruf arbeitete. Sie informierte die älteren Kollegen darüber, dass diese Didaktik an der Hochschule nicht mehr gelehrt würde und sehr alt ist. Diese Information ließ die Überzeugung der Gruppe etwas lockern. [...] Eine der älteren Lehrkräfte machte sich die Didaktik nach 
Hinweis: Diese Artikelfassung entspricht nicht vollständig dem in der Zeitschrift PADUA veröffentlichten Artikel unter https://doi.org/10.1024/1861-6186/a000579. Dies ist nicht die Originalversion des Artikels und kann daher nicht zur Zitierung herangezogen werden. Bitte verbreiten oder zitieren Sie diesen Artikel nicht ohne Zustimmung des Autors.

Schwarz-Govaers zu eigen und war von da an absolut begeistert von dieser und versuchte die Didaktik an alle Kollegen zu vermitteln. Durch ihre Begeisterung entschied sich das Team dann allgemein für diese Didaktik. Dies bereitete mir als Praktikantin etwas Bauchschmerzen, da mir persönlich die Didaktik von Darmann-Finck gut gefällt. Ich hatte die Befürchtung, dass keine andere Lehrkraft große Lust hatte, sich so gut mit einer anderen Didaktik auseinanderzusetzen, und sie deswegen sich aus Desinteresse für den leichtesten Weg entschieden hatten." (N_13)

\section{Interpretation}

Aufgrund der (pflege-)beruflichen und didaktisch-pädagogischen Grundsätze, die im Begründungsrahmen sowie in den Begleitmaterialien der Rahmenlehrpläne von der Fachkommission als gemeinsames Verständnis postuliert werden, erübrigen sich aus meiner Sicht Fragen, wie sie die Teilnehmenden der Curriculum-AG oben diskutieren. Auch wenn in den Rahmenlehrplänen keine Theorien, Modelle und Konzepte explizit benannt werden, so haben doch einige darin deutliche Spuren hinterlassen. Im Narrativ fällt auf, dass die Diskutierenden sich nicht an den Rahmenlehrplänen orientieren, sondern curriculare Entscheidungen über persönliche Erfahrungen treffen, die sie sowohl in der curricularen Arbeit als auch in der Unterrichtspraxis gesammelt hatten. Dieses Phänomen ist wohl zurückführbar auf die Wirksamkeit subjektiver Theorien, d.h. auf biografisch entstandene Gedanken, Gefühle und Gewohnheiten, mit denen Lehrende die eigene (Um-)Welt deuten und verstehen. Von diesen - in der Regel ausgeprägten und veränderungsresistenten - Theorien lassen sich Lehrende eher leiten, als neues Wissen in das bereits existierende Handlungsrepertoire zu integrieren. Selbst wenn sie neues Wissen - hier zu pflegedidaktischen Theorien - bereits erworben haben, lassen sie sich dadurch in ihrem Handeln kaum beeinflussen (Wahl 2013, S. 12). Sie kommen vom neu erworbenen Wissen erst dann zum zielgerichteten und bewussten Handeln, wenn sie aus der konkreten Handlung heraustreten können, um „mit hoher Reflexivität Schritt für Schritt das eigene Handeln zu ändern“ (Wahl 2013, S. 27). Oder sie reflektieren auf durchlebte Erfahrungen und kommen zu neuen Einsichten, d.h. durch die Reflexion von persönlichen Handlungen erwerben sie neues Wissen (Wahl 2013, S. 28).

In beiden Fällen sollten curriculare Entwicklungsprozesse „Menschen stärken [, um] die Sachen [zu] klären“ (von Hentig 1985). Lehrende der Curriculum-AG könnten das ,Neue‘ der Rahmenlehrpläne dadurch anschlussfähig machen, dass sie entweder die Auswirkungen des gemeinsamen Verständnisses der Fachkommission auf das bisherige Handeln reflektieren oder - ausgehend von ihren bewährten Unterrichten und im Abgleich mit dem ,Neuen“ - darüber nachdenken, was bleiben kann und was sich ändern muss, damit ein tatsächlich generalistisches Curriculum entsteht. 


\section{Drittes Verhinderungsmuster: Gegen Windmühlen kämpfen}

Entwickeln - je in der Alten-, Kranken- oder Kinderkrankenpflege sozialisierte und darüber hinaus im pädagogischen Selbstverständnis unterschiedlich geprägte Lehrende gemeinsam ein generalistisches Curriculum, äußern sie oft gegensätzliche Gefühle von Freude bis Angst. Dadurch kommt es zu Störungen, Spannungen und Widerständen. Weil manche Lehrende sich nicht darüber verständigen, wie mit den Rahmenlehrplänen umzugehen sei, führen sie entweder ergebnislose Debatten oder beteiligen sich gar nicht.

Im folgenden Narrativ konkretisieren sich entsprechende Beobachtungen:

„Die junge Lehrerin erzählte mir, dass sie sehr viel Potenzial in dem neuen Rahmenlehrplan sähe und auch hochmotiviert sei, die Generalistik anzugehen, jedoch gäbe es immer einzelne im Kollegium, die durch konservative „Das haben wir immer schon so gemacht"-Ansichten den Prozess aufhalten und blockieren würden. Dafür erstellte sie mehrere Flipcharts, welche den Prozess der CE darstellen sollten, und präsentierte diese in den Konferenzen für mehr Transparenz und zur Ermutigung stark skeptischer Kollegen. [...] Wenn ich in den Konferenzen saß, in denen sie den Prozess und die bestehenden Ergebnisse dem Kollegium vorstellte, zerbrach es mir das Herz, wenn ihre Arbeit und Energie auf Kommentare von älteren Kollegen wie „dafür haben wir eh keine Zeit", „ich habe das seit 40 Jahren so gemacht“ und „, ich werde jetzt nur noch die alten Klassen betreuen, damit ich das nicht machen muss“, stieß. Sie beschrieb mir ihre Gefühle, dass die Arbeit am Curriculum zwar sinnvoll wäre, aber dass sie im Team manchmal das Gefühl hätte, gegen Windmühlen zu kämpfen." (N_14)

\section{Interpretation}

Durch das zu lösende Problem der generalistischen Ausbildung sehen sich Lehrende - nicht immer gewollt - dazu veranlasst, sich mit ihrem bisherigen pflegerischen und pädagogischen Selbstverständnis auseinanderzusetzen. Ab sofort sollen sie generalistisch denken und handeln, sogar generalistisch ausbilden und dafür neue schulinterne Curricula nicht mehr nur aus der Perspektive „überzeugte[r] Altenpfleger*innen und Gesundheits- und (Kinder)Krankenpfleger*innen“ (Winter 2020, S. 151) entwickeln. Gerade aus dieser - eben nicht generalistischen - Perspektive sind die im Narrativ dargestellten Reaktionen einiger Lehrender durchaus nachvollziehbar. Ferner dominiert in der „pflegepädagogischen Praxis“ (Oelke i.E.) wohl bis heute der eher dozierende Lehrende, der zwar ein ganzheitliches Pflegeverständnis hat, aber - auch aufgrund bisheriger Lehrpläne - eher verrichtungsorientiertes Rezeptwissen vermittelt. Die aktuellen Veränderungen gefährden entsprechende Werte, Einstellungen sowie Gewohnheiten und führen zu Verlustängsten: Das haben wir immer schon so gemacht. Viele Lehrende suchen in den Rahmenlehrplänen nach ,ihren' Unterrichtsthemen, finden sie nicht (mehr) und stellen sich die berechtigte Frage, ob alles, was bislang gut war, künftig nicht mehr wichtig ist. Gleichwohl werden sie konfrontiert mit größeren 
Hinweis: Diese Artikelfassung entspricht nicht vollständig dem in der Zeitschrift PADUA veröffentlichten Artikel unter https://doi.org/10.1024/1861-6186/a000579. Dies ist nicht die Originalversion des Artikels und kann daher nicht zur Zitierung herangezogen werden. Bitte verbreiten oder zitieren Sie diesen Artikel nicht ohne Zustimmung des Autors.

Veränderungen als vermutet oder erhofft. Ihr Umgang mit anstehenden Veränderungsprozessen hängt ab von ihren Wahrnehmungen, Gefühlen und Deutungen, die damit aufgrund berufsbiografischer Lebenszusammenhänge verbunden sind. So könnten die oftmals ablehnenden Reaktionen Lehrender erklärbar sein. Gerade in beruflichen Umbruchsituationen stabilisiert das biografisch entstandene Selbstverständnis Kontinuität und Kohärenz. Lehrende bewerten demnach die Anstrengung beruflicher Herausforderungen mit der potenziellen Leistung des „biografische[n] Handlungsmotor[s]“ (Ostermann-Vogt 2011, S. 247) und machen damit die eigene Biografie sowohl zur Ressource als auch zur Barriere. Damit sie sich aber nun im generalistischen Spannungsfeld zurechtfinden, benötigen sie sowohl ein Umfeld, in dem sie auf bewährte Problemlösungsmuster zurückgreifen können, als auch Gelegenheiten dazu, sich auf ihre Haltungen zu besinnen (Ostermann-Vogt 2011, S. 250). Arbeiten sie nämlich nicht nur daran, ,von außen' an sie herangetragene Anforderungen umzusetzen, sondern gleichzeitig an eigenen Haltungen, können sie insofern identitätsstiftende Entwicklungsprozesse gestalten, als sie eigene berufliche Handlungs- und Deutungsmuster aufgreifen und das persönliche Selbstverständnis fortentwickeln. Erst in einem überzeugenden, ,selbstbildungsfreundlichen“ Umfeld entstehen bei der schulinternen Curriculumarbeit kreative Prozesse hin zu einer tatsächlich generalistischen Ausbildung. Für die Gestaltung curricularer Entwicklungsprozesse bleibt festzuhalten, dass aktuelle Veränderungen weder über den gemeinsamen Diskurs noch über ein festes Zeitkontingent angestoßen werden können. Vielmehr bedürfen sie der „pragmatische[n] Professionalität" (Terhart 2002, S. 102; in: Reh 2004, S. 359) im Sinne einer änderungsbefähigten Lernbereitschaft: Lehrende zeigen eine professionelle Haltung dadurch, dass sie - immer ausgehend von anstehenden biografischen Veränderungsnotwendigkeiten - an sich arbeiten und sich nicht als ,fertig' betrachten.

\section{Eine Anregung zur Curriculumentwicklung}

Im Januar 2020 reichte die Fachkommission Begleitmaterialien zu den Rahmenlehrplänen nach, um Lehrende an Pflegeschulen zum Diskurs „über die theoretischen Begründungen und ihre bildungspraktischen Implikationen“ (2020b, S. 4) anzuregen. Sie fordert sie dazu auf, curriculare Prozesse durch die Auseinandersetzung mit den „umrissenen Verständnisse[n]“ (2020b, S. 4) zu grundlegenden Begriffen voranzutreiben, um die eigene Schul- und Ausbildungskultur weiterzuentwickeln. In einem ersten Schritt schlägt sie ihnen vor, sich zu eigenen Überzeugungen auszutauschen: Lehrende sollen klären, welches Pflege-, Berufs-, Bildungs- und Lernverständnis sie vertreten. Zweitens sollen sie das lehrplanimmanente Verständnis mit dem eigenen Verständnis vergleichen, diskutieren und schließlich die Ergebnisse dieser Diskussion für eigene curriculare Prozesse nutzen. Diese Empfehlung dürfte schwierig umzusetzen sein. Denn die aus den „Gegenhorizonte[n]“ entstandenen „Ableitungen konkreter Konsequenzen“ (2020b, S. 4) sind nicht zielführend: Curriculare Einheiten der Rahmenlehrpläne basieren auf dem gemeinsamen Verständnis der Fachkommission, 
Hinweis: Diese Artikelfassung entspricht nicht vollständig dem in der Zeitschrift PADUA veröffentlichten Artikel unter https://doi.org/10.1024/1861-6186/a000579. Dies ist nicht die Originalversion des Artikels und kann daher nicht zur Zitierung herangezogen werden. Bitte verbreiten oder zitieren Sie diesen Artikel nicht ohne Zustimmung des Autors.

weshalb grundlegende Begriffe zwar diskutiert, aber wohl kaum in ihrem Kern verändert werden können.

Außerdem zeigt sich in den oben beschriebenen Verhinderungsmustern, dass Lehrende nicht auf einer rein kognitiven Ebene ansprechbar sind, wenn sie anspruchsvolle und schwierige Entwicklungsprozesse durchlaufen (sollen oder müssen). Vielmehr benötigen sie meiner Erfahrung nach vor allem einen emotionalen und pragmatischen Zugang, damit sie die Rahmenlehrpläne erleben und erfahren, curriculare Einheiten erarbeiten und - darüber hinaus - neue Unterrichte planen und durchführen können. Im folgenden Abschnitt wird eine Anregung skizziert, wie Lehrende sich durch die Auseinandersetzung mit Haltungen einen Zugang zu curricularen Einheiten bahnen könnten.

\section{Curriculumarbeit als Arbeit an und mit Haltungen}

Jeder Mensch hat eine Haltung. Sie ist einerseits etwas Individuelles und Persönliches und führt zurück auf prägende biografische Lebenszusammenhänge. Andererseits weisen Einzelhaltungen kollektive Gemeinsamkeiten auf, d.h. Menschen teilen mit anderen Menschen, mit denen sie etwas verbindet, einen ganz bestimmten Habitus, der unter anderem durch den Beruf geprägt wird. Dazu gehören bei Lehrenden die pflegeberufliche Sozialisation in der Alten-, Kranken- oder Kinderkrankenpflege sowie das pädagogische Selbstverständnis unterschiedlicher Lehrergenerationen.

Allerdings zeigen sich Haltungen - wie durch die oben aufgeführten Verhinderungsmuster bestätigt - oft als „eingeschliffene [unbewusste] Verhaltensmuster“ (Oelke, Scheller 2014, S. 8), die zwar erklärt, aber nicht voll begriffen werden können. Verarbeiten Lehrende emotionale Erlebnisse - wozu die curriculare Ausarbeitung generalistischer Unterrichte gehört - dadurch reflexiv, dass sie sich diese zu erklären versuchen, so dürften sie in diesen Erklärungen bewusste, aber auch unbewusste Vorstellungen und Wünsche inkludieren. Durch curriculare Lernprozesse könnten sie insbesondere dann in ihrer Identität gestärkt werden, wenn sie sowohl Wahrnehmungs-, Denk- und Deutungsmuster als auch ihre sozialen Handlungsmuster - also innere und äußere Haltungen - bearbeiteten.

In generalistischen Unterrichten müssen sie künftig wohl vieles anders durchführen, als es ihren Wünschen, Vorstellungen und Erwartungen entspricht, und sind folglich überfordert. Sie stehen vor der Aufgabe den generalistischen Unterricht aufgrund von „unklare[n], divergente[n] und widersprüchliche[n] Rollenanteile[n[“ (Gudjons, Wagener-Gudjons, Pieper 2008, S. 23) mit der (erneuten) Suche nach der eigenen Identität zu verknüpfen. Durch die biografische Selbstreflexion bereits bei der Curriculumentwicklung können sie dabei unterstützt werden, „Brücken zu schlagen“ (Gudjons, Wagener-Gudjons, Pieper 2008, S. 23) zwischen der Vergangenheit, der Gegenwart und der Zukunft. Dabei könnten sie folgende Fragen beantworten: 
Hinweis: Diese Artikelfassung entspricht nicht vollständig dem in der Zeitschrift PADUA veröffentlichten Artikel unter https://doi.org/10.1024/1861-6186/a000579. Dies ist nicht die Originalversion des Artikels und kann daher nicht zur Zitierung herangezogen werden. Bitte verbreiten oder zitieren Sie diesen Artikel nicht ohne Zustimmung des Autors.

- Welche Ereignisse oder Personen haben meine Wahrnehmungen, Deutungen und Gefühle im Umgang mit zu pflegenden Menschen ebenso wie mit Auszubildenden geprägt?

- Welche Anteile davon zeigen sich, wenn ich Auszubildende unterrichte?

- Was davon möchte bzw. sollte ich bewahren und was verändern?

\section{Curriculumarbeit szenisch gestalten}

Zuallererst sollten Lehrende die Kernaussagen der Rahmenlehrpläne und insbesondere deren Wirken innerhalb der curricularen Einheiten nachvollziehen können. Sie müssten dazu befähigt sein, die Lehrpläne ,zwischen den Zeilen zu lesen‘, d.h. curriculare Einheiten hermeneutisch zu erschließen. So könnten sie unter anderem darüber nachdenken, (erstens) wie sie Kompetenzen einerseits von Bildungszielen unterscheiden und andererseits zusammen in der curricularen Einheit konkretisieren können und (zweitens) welche Pflege- und Unterrichtserfahrungen sie selbst mit den ,Themen' der curricularen Einheiten situativ verbinden. Dann erst sollten sie aus den Situationsmerkmalen der Rahmenlehrpläne konkrete Lernsituationen erarbeiten.

Von den begleiteten und von mir ,narrativisch' erinnerten Prozessen, in denen die oben skizzierten Verhinderungsmuster ebenfalls identifizierbar sind, lässt sich lernen, dass gerade die Arbeit Lehrender an und mit Haltungen eine wichtige Funktion hat: Curriculumentwicklung wird zur identitätsstiftenden Gelegenheit. Lehrende machen sich an die Weiterentwicklung des eigenen professionellen Selbstverständnisses, indem sie in (biografisch entstandenen), Szenen' relevante Unterrichts- und Pflegesituationen sowie darin gezeigte Haltungen, Handlungen, Interaktionen und Gefühle mit unterschiedlichen Verfahren des Szenischen Spiels ent- bzw. aufdecken und - gleichzeitig - nachdenken über mögliche Auswirkungen dieser ,Szenen' auf künftige generalistische Unterrichte. Heterogen sozialisierte Kollegien sammeln besonders wertvolle und hilfreiche Erfahrungen, wenn sie beispielsweise Standbilder zu Erfahrungen mit zu pflegenden Menschen aller Altersgruppen in unterschiedlichen Kontexten erkunden oder ,Unterrichtsbilder' aus der Alten-, Kranken- oder Kinderkrankenpflege deuten. Szenische Arbeit und hermeneutische Erschließung der Rahmenlehrpläne stehen in einem wechselseitigen Zusammenhang, sodass nach und nach anschlussfähige Lernsituationen entstehen, durch die sowohl das grundlegende Verständnis der Fachkommission als auch die Erfahrungen Lehrender auf gleicher Ebene abgebildet werden können. 
Hinweis: Diese Artikelfassung entspricht nicht vollständig dem in der Zeitschrift PADUA veröffentlichten Artikel unter https://doi.org/10.1024/1861-6186/a000579. Dies ist nicht die Originalversion des Artikels und kann daher nicht zur Zitierung herangezogen werden. Bitte verbreiten oder zitieren Sie diesen Artikel nicht ohne Zustimmung des Autors.

\section{Fazit}

Abschließend sei Brechts ,Haltung' wenigstens angedeutet, die den pflegedidaktischen Ansatz des szenischen Spiels prägt und darüber hinaus aktuelle curriculare Entwicklungsprozesse leiten könnte:

„Unsere Haltung kommt von unseren Handlungen, unsere Handlungen kommen von der Not! Wenn die Not geordnet ist, woher kommen dann unsere Handlungen? Wenn die Not geordnet ist, kommen unsere Handlungen von unserer Haltung!“ (Brecht, zit. nach Steinweg 1976, 47, in Oelke, Scheller 2014, 9).

Machen Lehrende in einem anspruchsvollen Prozess eigene und fremde Haltungen sichtbar, um curriculare Freiräume zu erkennen, legitimieren sie ein entstehendes Curriculum, in dem sie zwar gesetzte Standards umsetzen, aber nicht aufgeben, was gut war und sich bewährt hat. Mögen sie auch zukünftig für einen neuen Beruf ausbilden: Der Kern von Pflege (Friesacher 2015) verändert sich nicht. Curriculare Prozesse kontrollierend (nicht umgekehrt) gelangen sie - nach und nach - zu anderen Haltungen.

\section{Literatur}

Fachkommission nach § 53 Pflegeberufegesetz (2020a). Rahmenpläne der Fachkommission nach § 53 PflBG. Zugriff am 29.08.2020 unter https://www.bibb.de/dokumente/pdf/geschst_pflgb_rahmenplaene-der-fachkommission.pdf

Fachkommission nach § 53 Pflegeberufegesetz (2020b). Begleitmaterialien zu den Rahmenplänen der Fachkommission nach § 53 PfIBG. Zugriff am 29.08.2020 unter https://www.bibb.de/dokumente/pdf/Begleitmaterialien_zu_den_Rahmenplaenen.pdf

Friesacher, H. (2015). Wider die Abwertung der eigentlichen Pflege. Intensiv 4/2015, S. 200-214.

Gudjons, H., Wagener-Gudjons, B. \& Pieper, M. (2008). Auf meinen Spuren: Übungen zur Biografiearbeit. Bad Heilbrunn: Klinkhardt.

Göhlich, M. (2008). Schulentwicklung als Machbarkeitsvision. Eine Re-Vision im Horizont professioneller Ungewissheit. In W. Helsper, S. Busse, M. Hummrich \& R.-T. Kramer (Hrsg.), Pädagogische Professionalität in Organisationen (S. 263-275). Wiesbaden: VS.

Hentig, H. von (1985). Die Menschen stärken, die Sachen klären. Stuttgart: Reclam.

Nünning, V. (2011/2012). Narrativität als interdisziplinäre Schlüsselkategorie. Auszug aus dem Jahresbericht „Marsilius-Kolleg“. Zugriff am 29.08.2020 unter https://www.marsilius-kolleg.uni-heidelberg.de/md/einrichtungen/_ mk/publikationen/mk_jb_05_narrativitaet_als_interdisziplinaere_schluesselkategorie.pdf

Oelke, U. (i.E). Erfahrungsbezogenes Lernen in den Gesundheitsfachberufen. In K.-H. Sahmel, I. Darmann-Finck (Hrsg). Pädagogik im Gesundheitswesen. Berlin: Springer.

Oelke, U., Scheller, I. (2014). Grundsätze erfahrungsbezogenen bzw. szenischen Lernens. Berlin: Cornelsen. Zugriff am 26.06.2019 unter https://www.cornelsen.de/herausforderungpflege/1.c.3511709.de

Ostermann-Vogt, B. (2011). Biographisches Lernen und Professionalitätsentwicklung: Lernprozesse von Lehrenden in Pflegeberufen. Wiesbaden: VS.

Panke-Kochinke, B. (2016). Let's work together: Schulentwicklung in der beruflichen Ausbildung an Pflegeschulen. Frankfurt/M.: Mabuse.

Reh, S. (2004). Abschied von der Profession, von Professionalität oder vom Professionellen? Theorien und Forschungen zur Lehrerprofessionalität. Zeitschrift für Pädagogik 50 (3), S. 358-372. 
Hinweis: Diese Artikelfassung entspricht nicht vollständig dem in der Zeitschrift PADUA veröffentlichten Artikel unter https://doi.org/10.1024/1861-6186/a000579. Dies ist nicht die Originalversion des Artikels und kann daher nicht zur Zitierung herangezogen werden. Bitte verbreiten oder zitieren Sie diesen Artikel nicht ohne Zustimmung des Autors.

Wahl, D. (2013). Lernumgebungen erfolgreich gestalten: Vom trägen Wissen zum kompetenten Handeln. Bad Heilbrunn: Klinkhardt.

Winter, C. (2020). Generalistische Pflege auf Augenhöhe. In O. Dibelius, G. Piechotta-Henze (Hrsg.), Menschenrechtsbasierte Pflege (S. 149-159). Bern: Hogrefe.

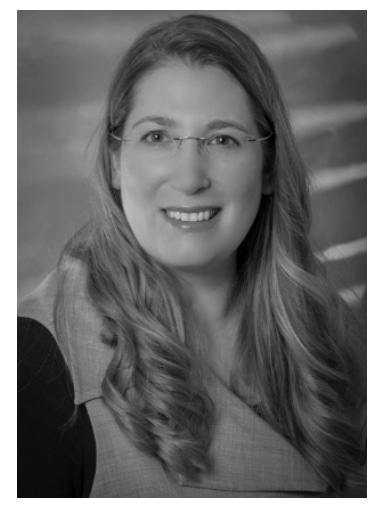

Prof. Dr. phil. Claudia Winter

Professorin für Gesundheits- und Pflegepädagogik, insbesondere Pflegepraxis und Pflegedidaktik

Evangelische Hochschule Nürnberg

E-Mail: claudia.winter@evhn.de 